\title{
Die Magensaftsecretion des Neugeborenen.
}

Von

Otto Cohnheim und Franz Soetbeer.

(Aus dem physiologischen Institut und der Kinderklinik zu Heidelberg.)

(Der Redaction zugegangen am 23. Februar 1903.)

Die letzten Jahre haben uns auf dem Gebiete der Verdauungslehre hochbedeutsame Fortschritte gebracht. Während man sich bisher hauptsächlich mit den verdauenden Fermenten beschäftigte, hat uns $\mathrm{Pawlow}$ gezeigt, welche verwickelten nervösen Vorgänge sich abspielen müssen, damit die richtige Coordination der Verdauung zu Stande kommt. Es genügt nicht, dass mit der Nahrung verdauliche Stoffe dem Körper überhaupt zugeführt werden, sondern es kommt sehr viel darauf an, wie sie genossen werden. Die Verdauung arbeitet nur dann absolut zweckmässig, wenn durch den Act der Aufnahme die Sinne des Thieres in der geeigneten Weise erregt werden.

Es musste daher von besonderem Interesse sein, die Magenverdauung $z u$ einer Zeit $z u$ untersuchen, in der die Art der Nahrungsaufnahme eine völlig abweichende ist, beim Neugeborenen. Denn erstens ist der Vorgang der Nahrungszufuhr an sich abweichend: das junge Säugethier isst, trinkt und kaut nicht, sondern saugt; zweitens erhält es eine ganz andere und besonders angepasste Nahrung, die Milch. Drittens ist ein Theil der Sinnesorgane noch unentwickelt, indem Geruch und Geschmack anscheinend schon da sind, junge Hündchen dagegen noch nicht sehen. Und endlich fehlen dem Neu- 
geborenen alle Erfahrungen, auf Grund deren es lernen und nach denen es sein Handeln zweckmässig gestalten kann. ${ }^{1}$ )

Das erwachsene Thier secernirt Magensaft, wenn es eine wohlschmeckende Speise sieht, riecht oder schmeckt, gleichgültig, ob die Speise wirklich in den Magen gelangt oder nicht. Ist dieser Vorgang bei den mangelhaft entwickelten Sinnesorganen des Kopfes beim Säugling schon vorhanden? Wenn ja, durch welches Sinnesorgan wird er ausgelöst? Secernirt endlich der Neugeborene überhaupt schon Magensaft? Und wie ist er zusammengesetzt?

Diese Fragen schienen uns von mehreren Gesichtspunkten der Untersuchung werth. Erstens natürlich wegen der Bedeutung für die Physiologie und die Praxis der kindlichen Ernährung und Verdauung. Zweitens aber konnten wir so das Wesen des "psychischen Magensaftes» klarer erkennen. Es liegen ja offenbar zwei Möglichkeiten vor: entweder bringen die Thiere die Fähigkeit, den für die aufgenommene Nahrung passenden Verdauungssaft zu secerniren, als Reflex, der von Auge und Nase durch Gehirn und Vagus zum Magen läuft, schon mit auf die Welt, oder aber es beruht die durch Sinnesreize erregte zweckmässige Secretion auf Urtheilsbildung auf Grund von Erfahrungen, die das Individuum im Laufe des Lebens sammelt. Denn es ist ja möglich, dass die Erregung des Gesichts-, des Geruchs- und Geschmackssinnes gar nicht ohne Weiteres zur Hervorrufung des psychischen Magensaftes ausreicht. Die primären Eindrücke dieser Sinnesorgane verlangen vielleicht vor ihrer Uebertragung auf die Verdauungsorgane noch immer eine gewisse Urtheilsbildung, einen höheren psychischen Vorgang, wie das ja die Bezeichnungen "Appetitsaft oder psychischer Magensaft» ausdrücken würden. Wenn ich einem Hunde ein Stück Fleisch vorhalte und er bildet Appetitsaft, der beim Anbieten eines Kieselsteins ausbleibt, so kann darin ein erlernter psychischer Vorgang, die Unterschei-

1) Es wird uns hoffentlich Niemand verdenken, wenn wir die geläufigen «subjectiven * Ausdrücke beibehalten. 
dung von Geniessbarem und Ungeniessbarem, enthalten sein. Es läge dafür eine Analogie vor in Pawlow's Beobachtungen über Speichelsecretion, ${ }^{1}$ ) nach denen Verdünnungsspeichel zunächst nur auf Reizung der Mundhöhle secernirt wird. Hat man den Reflex aber einige Male ausgelöst, so genügt es, den Versuch vor den Augen der Thiere vorzubereiten, um Erinnerungen in ihnen zu wecken und den Speichel fliessen $z u$ lassen. Dazu gehört sicher ein complicirter psychischer Vorgang. Ob aber angeborene Reflexe vorhanden sind, lässt sich am einfachsten durch Versuche am Neugeborenen entscheiden.

Früher ist die Frage, ob das Saugen an sich, beziehentlich das Verlangen nach Speise beim menschlichen Säugling Magensaftabsonderung hervorruft, schon einmal gestellt worden von Bauer und Deutsch. ${ }^{2}$ ) Sie schreiben: "Führt man das Saugfläschchen an den Mund des - scil. hungernden - Säuglings, so hascht dieser darnach und bewegt die Lippen, als würde er schon saugen. Die sofort angestellte Untersuchung des Magensaftes ergab gänzlichen Mangel an Säure oder eine minimale, dem Einfluss der Sonde zumessbare Menge derselben. An eine solcherart provocirbare reflectorische Secretion kann also nicht gedacht werden.» Da wir nun heute wissen, dass Sondiren keine Secretion hervorruft, und dass eine Latenzzeit von 5-6 Minuten besteht, kann die Schlussfolgerung wohl kaum als zwingend angesehen werden. Sonst sind wir nur über den Fermentgehalt der Verdauungsdrüsen in den ersten Lebenswochen durch die Untersuchungen von Zweifel, ${ }^{3}$ ) Hammarsten ${ }^{4}$ ) und Gmelin ${ }^{5}$ ) unterrichtet. Dar-

1) S. G. Wulfson, Dissertat., St. Petersburg, Maly's Jahresber. Bd. 29, S. 361, 1899. - L. Tolotschinoff, Versamml. nordischer Naturforscher u. Aerzte zu Helsingfors, 1902. Sect. f. Anatom., Physiol. u. medic. Chem., S. 42. - Vergl. auch Münch. medic. Wochenschr. 1902, S. 2173.

2) L. Bauer u. E. Deutsch, Jahrbuch für Kinderheilkunde 48, S. 22, 1898 , S. 27.

3) P. Zweifel, Untersuchungen über den Verdauungsapparat des Neugeborenen. Berlin 1874.

4) O. Hammarsten, Beiträge zur Anatomie u. Physiol., G. Ludwig zum 60. Geburtstag gewidmet, 1875.

5) W. Gmelin, Pflüger's Arch., 90, 590, 1902. 
nach enthält der Magen des menschlichen Säuglings șchon bei der Geburt Pepsin und Lab, neugeborene Hunde dagegen produciren die beiden Fermente erst von der dritten Woche ab. Aber die Angaben Gmelin's lassen es zweifelhaft erscheinen, ob neugeborene Hunde überhaupt schon Magensaft secerniren, da er in ihren Mägen immer nur Milchsäure fand, dagegen zu keiner Zeit freie Salzsäure, die doch neben den Fermenten das Secret der Magendrüsen auszeichnet. Auch diese Frage liess sich am leichtesten entscheiden, wenn man ganz junge Hündchen mit Pawlow's Methodik untersuchte.

Wir haben unsere Beobachtungen an jungen Hündchen gemacht und zwar von drei verschiedenen Würfen, die wir kurz hintereinander beobachteten. Die des ersten haben wir erst im Alter von etwa 14 Tagen operirt, da wir Anfangs die Schwierigkeiten der Operationen überschätzt und uns gescheut hatten, an noch kleineren Thieren zu operiren. Wir verwendeten erst eine kleine Magenkanüle nach dem $\mathrm{Pawlow}$ 'schen Modell, ${ }^{1}$ ) doch erwies sich dieselbe bei den sehr kleinen Verhältnissen und der Zerreissbarkeit der Magenwand als ungeeignet, und wir vereinigten dann den Magen mit der Bauchwand mit einem kleinen Murphyknopf, der etwa 3 Tage hielt. Am bequemsten erwies sich schliesslich ein kleines Instrument, das der eine von uns $^{2}$ ) früher zu Versuchen über die Peritonealresorption benutzt hatte, und das in einer kleinen Kanüle besteht, um die die Magen- und die Bauchwand beide mit einer Tabaksbeutelnaht zusammengezogen und durch eine aufschraubbare Platte festgepresst wurden. Zunächst glaubten wir, mit der Anlegung einer Magenfistel allein auskommen zu können, und liessen die so operirten Thierchen - als Ersatz der Scheinfütterung - an den Zitzen einer trächtigen Hündin saugen, die noch keine Milch und auch noch kein Colostrum lieferte. Dabei verschluckten die Thierchen aber so viel Speichel, dass wir keinen reinen Magensaft und in Folge dessen wider-

1) J. P. Pawlow, Physiol. Chirurgie d. Verdauungskanals. Ergebnisse d. Physiol. v. Asher u. Spiro, I, 1, S. 254.1902.

2) O. Cohnheim, Ueber die Resorption im Dünndarm und der Bauchhöhle, Zeitschr. f. Biol. 37, 443, 1898. S. 467. 
sprechende Resultate erhielten, deshalb führten wir am Tage darauf noch die Oesophagotomie aus. Zwei derartig operirte Hündchen haben wir vom 18. Lebenstage an mehrere Tage beobachtet.

An die Zitzen der Mutter angelegt, begannen sie sofort kräftig zu saugen, und wir fanden dann im Magen eine geringe Menge einer stark sauren Flüssigkeit, die mit dem Günzburg'schen Reagens sowie mit Tropaeolin die schönsten positiven Reactionen gab, also freie Salzsäure enthielt. In einem Falle erhielten wir soviel Secret, dass wir Verdauungsversuche damit machen konnten. Die 1-2 ccm, die aus der Magencanüle entleert werden konnten, wurden mit Salzsäure verdünnt: sie lösten eine Fibrinflocke und brachten, nach vorheriger Neutralisation mit Baryumcarbonat, ${ }^{1}$ ) Hunde- und Kuhmilch zum Gerinnen. Die Flüssigkeit bestand also zweifellos aus Magensaft.

Wir haben dann die beiden Thierchen ausser an ihrer milchgebenden Mutter auch wieder an der trächtigen Hündin saugen lassen, deren Brustdrüsen weder Milch noch Colostrum abgaben. Mit dem gleichen Erfolg: auch jetzt wurde Magensaft secernirt. - An beiden Thierchen haben wir die Versuche, nach vorheriger gründlicher Ausspülung mit warmem Wasser, oft wiederholt, ausnahmslos mit positivem Erfolg.

Damit war zunächst entschieden, dass Hündchen im Alter von 18 Tagen salzsäurehaltigen Magensaft secerniren; aber die gegentheiligen Angaben Gmelin's sind erklärlich. Denn als wir den Thierchen mittelst eines Katheters Kuhmilch oder Hundemilch in den Magen einbrachten, fanden wir $1^{1 / 2}$ Stunden später im Magen das Casein geronnen und die Flüssigkeit stark sauer, dagegen keine freie Salzsäure, die offenbar durch die Eiweisskörper der Milch, wohl auch durch deren Salze - Hundemilch reagirt auf Lackmus stark alkalisch - neutralisirt, wie man beim Magen gewöhnlich sagt, gebunden worden ist. Dass nun aus dem Milchzucker Milch-

1) Pawlow schreibt zur Prüfung auf Labferment die Neutralisation mit Baryumcarbonat an Stelle des häufig gebrauchten Calciumcarbonats vor, weil nur dadurch mit Sicherheit alkalische Reaction vermieden werden könne. 
säure entstand, nimmt nicht Wunder. Wir haben uns ausdrücklich davon überzeugt, dass auch erwachsene Hunde 11/2. Stunden nach Milchfütterung keine freie Salzsäure im Mageninhalt zeigen, wobei noch an die von Pawlow entdeckte Hemmung der Magensaftsecretion durch das Milchfett erinnert sei. - Dass der Salzsäuregehalt des Magensaftes bisher übersehen worden ist, bildet einen neuen Beweis dafür, wie unbedingt erforderlich die Untersuchung der reinen Secrete ist.

Ausserdem aber ergab sich, dass saugende Thierchen schon "psychischen Magensaft» secerniren und dass ausserdem hierzu nicht einmal die Nahrung selbst erforderlich ist. Das Saugen an der Brustdrüse an sich bewirkt vielmehr bereits die Erregung der Magendrüsen. Ob dies mit Hülfe von Geruchsempfindungen, durch den Geruch der säugenden Mutter geschieht, oder ob etwa die Bewegung des Saugens schon als auslösender Reiz wirkt, haben wir nicht feststellen können, da wir die Hündchen nicht dazu bekamen, an einem Gummizulp oder unserem Finger zu saugen. Doch kann, in diesem Alter wenigstens, auch der Geschmack und Geruch der Nahrung schon reizend wirken, denn als wir das eine Hündchen aus einer Schale Milch lecken liessen, secernirte es Magensaft. In einem Falle versuchten wir auch die Latenzzeit der Saftsecretion zu bestimmen und fanden, dass saure Reaction zuerst nachweisbar war etwa 7 Minuten, nachdem der Hund die Zitzen der Mutter erfasst hatte.

Bei den Hündchen eines zweiten Wurfes versuchten wir nun die Operationen schon früher, am 4. Lebenstage, auszuführen, bei denen eines dritten bereits am 1. Tage. Hier stiessen wir aber auf eine unüberwindliche Schwierigkeit. Die Leber ist um diese Zeit noch so gross, dass sie den Magen völlig verdeckt. Es gelang uns nur bei einem 4 Tage alten Thierchen, den Magen zu erreichen, und dies starb einige Stunden nach der Operation. Bei den andern haben wir uns mit der Oesophagotomie begnügt. Aber die Durchtrennung der Speiseröhre ist ja auch die wichtigere von beiden Operationen, da sie den Magen isolirt, und da es durch Eingehen mit einem Nélaton-Katheter in die untere Oeffnung der Speiseröhre ganz leicht gelingt, in den Magen einzudringen und 
etwaigen Inhalt $\mathrm{zu}$ aspiriren. Die Oesophagotomie ist auch bei diesen kleinen Thierchen leicht, und wir konnten eines der am 4. Tage operirten Thiere mehrere Tage am Leben erhalten, und von den am 1. Tage operirten zwei Hündchen wenigstens das eine 24 Stunden beobachten, ehe wir es tödteten.

Wir stellten auf diese Weise fest, dass auch in dieser Zeit die Thierchen beim Saugen an den Zitzen der Mutter schon Magensaft secernirten, und dass dieser Magensaft freie Salzsäure enthält. Auch diese Versuche haben wir oftmals, und stets mit gleichem Erfolg wiederholt. Da wir die wenigen Tropfen stets für die Salzsäureprüfung verwendeten, konnten wir eine Fermentuntersuchung leider nicht vornehmen. Bei dem 4 tägigen Hündchen wurde Magensaft bereits beim Saugen an einer trächtigen Hündin ohne Milch abgesondert, bei dem neugeborenen konnten wir es nicht versuchen.

Neugeborene Hunde secerniren also schon am 1. Lebenstage "psychischen Magensaft». Leider ist es uns nicht gelungen, die Thierchen $\mathrm{zu}$ operiren, bevor sie überhaupt gesogen hatten. Unsere 3. Hündin warf Nachts, während es erst Morgens bemerkt wurde. Es können in maximo 9 Stunden vergangen sein, während deren die Hündchen auch wirklich gesogen haben, da sich wenigstens im Magen von zweien geronnene Milch vorfand. Indessen dürfte es wohl recht unwahrscheinlich sein, dass in dieser kurzen Zeit bereits Erfahrungen gesammelt worden sind. Es kann vielmehr keinem Zweifel unterliegen, dass die Erregung der Secretion des Magensaftes von den Receptionsorganen des Kopfes ein angeborener Reflex ist. Damit ist die eine im Eingang gestellte Frage beantwortet: Der Appetitsaft ist nicht der Ausdruck von im individuellen Leben gesammelter Erfahrung, sondern die Neugeborenen bringen ihn so gut mit auf die Welt, wie den Saugreflex.

Die nervöse Coordination der Verdauung ist also anscheinend eine ebenso fundamentale Nothwendigkeit zur Erhaltung des Lebens wie die Secretion von Verdauungssäften überhaupt und die Aufnahme von Nahrung. Welche Organe als Receptionsorgane für die Sinneseindrücke beim Neugeborenen in Wirksamkeit treten, haben wir eindeutig, wie gesagt, nicht. 
entscheiden können; denn durch das Anlegen an die trächtige Hündin können wir den Geschmackssinn ausschliessen, aber keine Entscheidung treffen zwischen dem Geruchsorgan und der Bewegung des Saugens.

Sicher ist ausserdem festgestellt, dass der secernirte Magensaft als wirksame Componente nicht Milchsäure, wie behauptet, sondern Salzsäure enthält. Auf Pepsin und Lab haben wir bei den allerjüngsten Thieren freilich nicht prüfen können, Schleimhautextracte wirkten so wenig, wie es Hammarsten und Gmelin gefunden haben, was seit Weinland's ${ }^{1}$ ) Entdeckung des Antipepsins nichts beweisen kann.

Für dieBeurtheilung der Verdauungsthätigkeit des menschlichen Säuglings geben die vorliegenden Experimente eine exacte Unterlage.

Sie zeigen uns, dass der Saugact an sich schon einen integrirenden Bestandtheil der Verdauungsarbeit bildet. Es ist sicher, dass die chemische und physikalische Beschaffenheit der Muttermilch und die Art der Aufnahme dieser Nahrung durch intensive Saugarbeit an der Mutterbrust das Optimum der Verdauung bei Optimum der Nahrung repräsentirt, gleichgiltig, welcher von beiden Reizen entscheidender ist. Sollte es gelingen, experimentell auch an menschlichen Säuglingen die Differenzen von psychischer Saftsecretion bei Saugen an der Mutter und an den üblichen Gummisaugern festzustellen, so würde vielleicht auch praktisch das Sauggeschäft bei künstlicher Ernährung so gestaltet werden können, dass die psychische Secretion ebenso günstig und reichlich functionirte wie bei der Ernährung an der Brust. Leider liegen solche Untersuchungen noch im weiten Felde, denn es ist unmöglich, bei ihnen das anzuwenden, was uns den Erfolg bei den jungen Thieren sicherte, die geniale Versuchsanordnung Pawlow's.

1) E. Weinland, Zeitschr. f. Biol., 44, 45, 1902. 